\title{
A Multi-frequency Catalog of Magnetic CVs
}

\author{
Paul A. Mason ${ }^{* \dagger}$ \\ New Mexico State University, Las Cruces, NM, 88003, USA \\ Picture Rocks Observatory and Astrobiology Research Center, \\ 1025 S. Solano, Suite D., Las Cruces, NM, 88001, USA \\ E-mail: pmason@nmsu.edu
}

\section{Lorena C. Monroy}

Picture Rocks Observatory and Astrobiology Research Center, 1025 S. Solano Dr., Las Cruces, NM, 88001 USA

\section{Natalie K. Wells}

Picture Rocks Observatory and Astrobiology Research Center, 1025 S. Solano Dr., Las Cruces, NM, 88001 USA

\section{Joshua B. Santana}

Picture Rocks Observatory and Astrobiology Research Center, 1025 S. Solano Dr., Las Cruces, NM, 88001 USA

\begin{abstract}
We present a new multi-frequency catalog of polars and intermediate polars (IPs) called MCVCatalog-1.0. The need for a new catalog of Magnetic Cataclysmic Variables (MCVs) is becoming increasingly important. The number of known and suspected cataclysmic variables (CVs) has doubled in just the last few years. It is expected to balloon by another factor of 10 in the next decade or so as several optical surveys, like LSST, take place. By establishing a catalog containing only magnetic CVs, i.e. MVCs, we have the freedom to tailor the catalog to a relatively homogeneous set of binaries. Along with cataloging the discovery of many new binaries, MCV-Catalog will keep track of poorly observed systems. Many of the most important astrophysical problems associated with accretion-binary astrophysics require the long-term study of a large sample of binaries. Multi-frequency and even multi-messenger observations are vital in developing our understanding of the interplay between gravity, angular momentum, magnetic fields, and fluid flow, characteristic of MCVs. Many studies focus on the hot new discoveries of albeit interesting binaries. By necessity, priorities are made and many binaries remain known but poorly studied. For this reason we find that MCV-catalog is a useful observing tool, and as a starting point for multi-frequency studies of MCVs. We anticipate that the MCV-catalog, established maintained by Picture Rocks Observatory, will be a 'living' catalog, beginning with all confirmed MCVs by January, 2020. New binaries will be added as they are reported and new data will be periodically added as they become available. Here we present a brief summary of the MCV-Catalog contents.
\end{abstract}

Multifrequency Behavior of High Energy Cosmic Sources - XIII - MULTIF2019

3-8 June 2019

Palermo, Italy 


\section{MCV-Catalog Link}

We present a new online catalog of Magnetic Cataclysmic Variables (MCVs), MCV-Catalog v.1.0. The catalog may be reached at the following link*:

https://www.picturerocksobservatory.org/mcv-catalog.

*We appreciate and encourage comments or suggestions that may be emailed or submitted through the website.

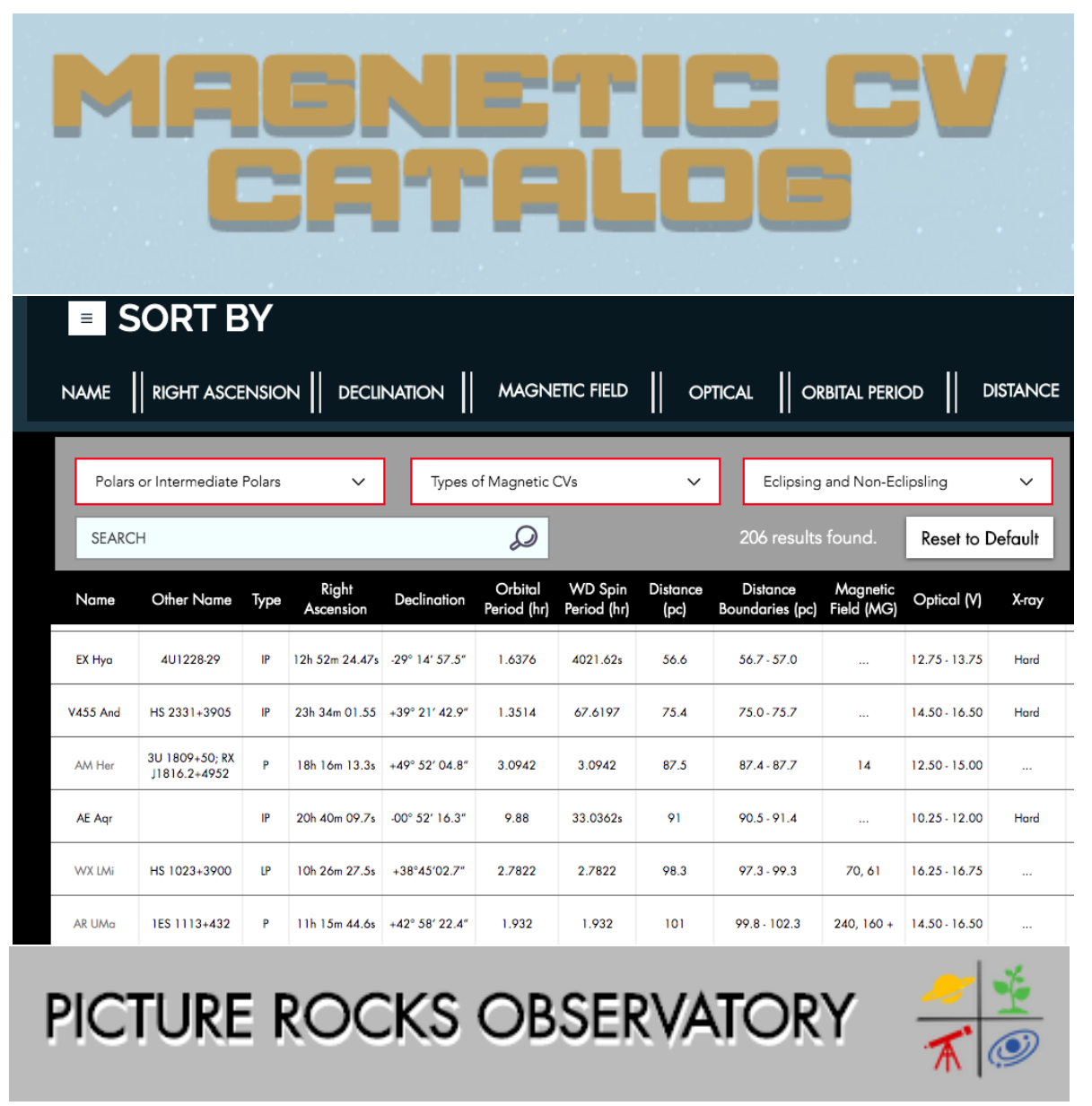

Figure 1: The MCV-Catalog main page contains the object list. The catalog is searchable and sortable. A detailed information page, including finder chart, is linked to each object.

\section{Introduction}

MCVs are close binaries containing a magnetic white dwarf accreting from a Roche-Lobe filling lower main sequence companion. The aim this catalog is provide a database of information

\footnotetext{
*Speaker.

${ }^{\dagger}$ This work supported by PRO-ARC
} 
on MCVs that begins with the snapshot of the field at the start of the 2020s and to continue to maintain and grow the catalog as the number of binaries increases into the future. Restricting the catalog to MCVs rather than to all CVs is done in order to focus content on specific properties of a reasonably homogeneous class of binaries. In this manner, we have first established a census of confirmed MCVs. In this first version of this catalog, we do no include candidate MCVs. However, the MCV-Catalog, see Figure 1 will be a living catalog with frequent updates.

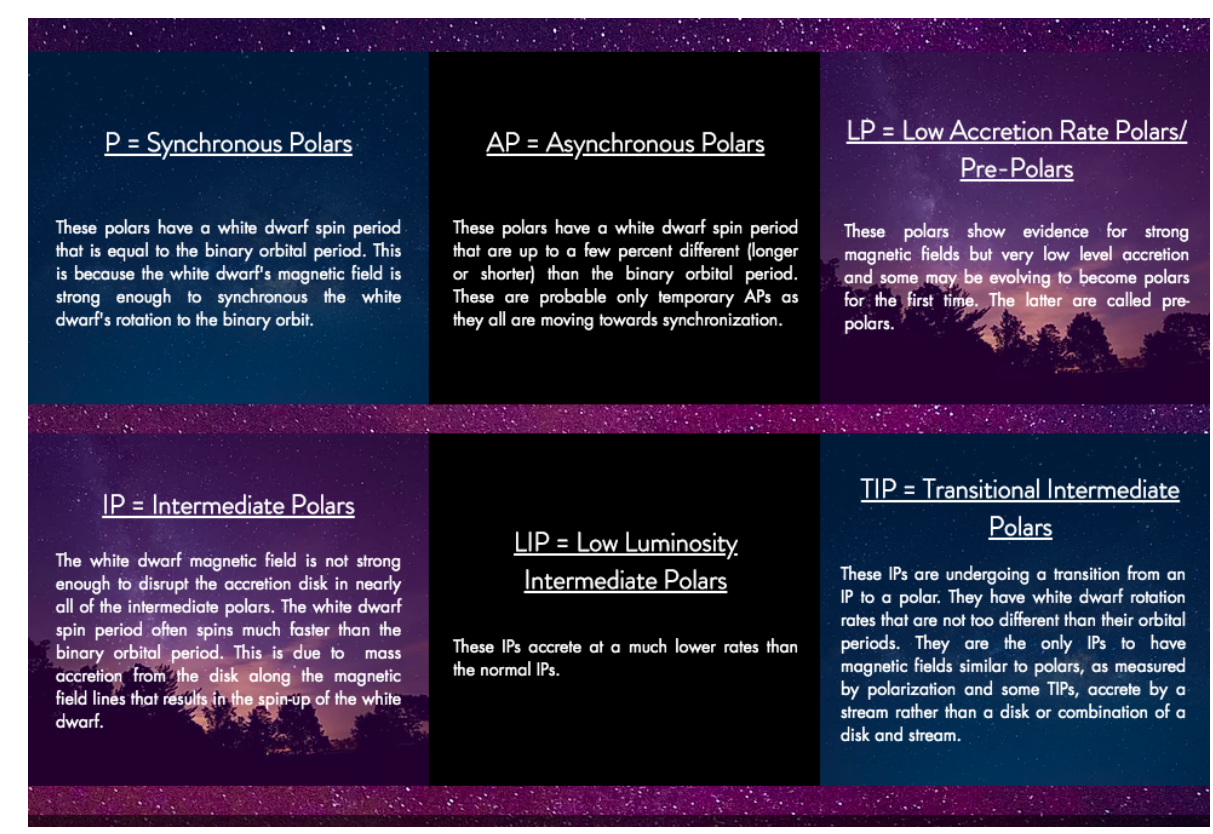

Figure 2: A snapshot from the MCV-Catalog website showing the definitions and classification system used.

Each binary is classified either as a Polar (P) or as an Intermediate Polar (IP) in a mutually exclusive manner. To illustrate the information given on the MCV-Catalog website, definitions used in our classification scheme are shown in Figure 2. Starting with polars, MCVs with white dwarfs magnetic fields $(B>1 \mathrm{MG})$ that most typically have two effects. All polars prevent the formation of an accretion disk, as matter is transferred from the donor through the inner Lagrangian point is directed to the surface of the white dwarf by the magnetic field. And most polars have white dwarf spin periods that are synchronized with the binary orbital period. In close binaries with periods less than 1-2 days, tidal forces act on the companion and cause the donor star's rotation to quickly become synchronous with the binary orbital period. The white dwarf's in polars are forced into synchronous rotation to the binary period, due to magnetic field interaction with the donor star. Some polars however are slightly asynchronous. These we call the asynchronous polars (APs). Referring to Table 1, we find that $4 \%$ of polars in the MCVs-Catalog are APs.

Intermediate polar (IP) classification is much more problematic than polar classification. IPs are a more heterogeneous group than polars. Here we follow the selection of confirmed and probable IPs from the NASA, IP Homepage of Koji Mukai, where binaries are given 1 to 5 stars based on their likelihood as IPs. We have selected only those with 3 or more stars and any new IP discoveries.

The catalog has two levels, first is the data table and second (linked the data table) is an 


\section{MAGNETIC CATACLYSMIC VARIABLES (CVs)}

Picture Rocks Observatory and Astrobiology Research Center

\section{AM Her \\ Long Period Polar}

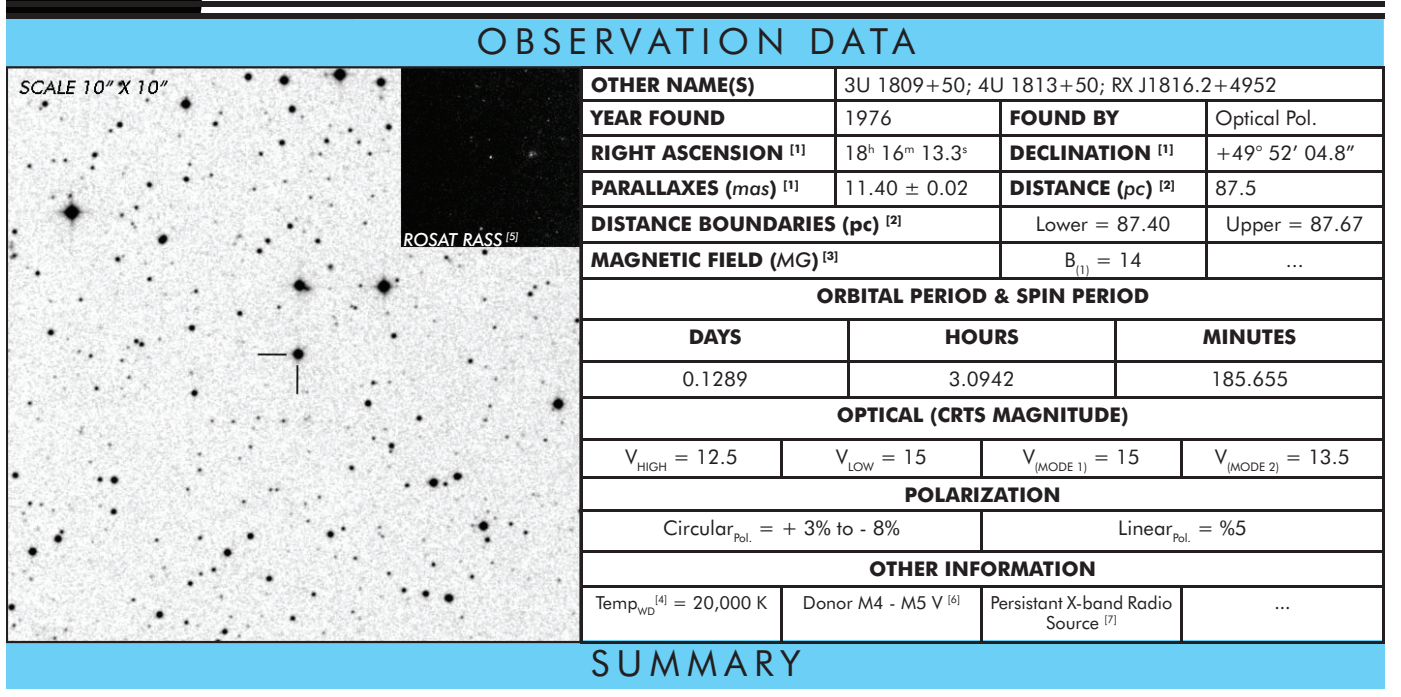

AM Herculis was identified, by Wolf (1924) as an irregular variable star ranging from 12-mag to 14-mag. It became the first discovered polar when Tapia (1977) followed up on the discovery of the X-ray source, $3 U 1809+50$, at 2 to $6 \mathrm{keV}$ by Giacconni et al. (1974) and in soff X-rays ( 0.15 to 0.28 keV) by Hearn, Richardson, \& Clark (1976). Tapia found a maximum linear polarization ( $V$ and I bands) of about $5 \%$ and circular polarization from $+3 \%$ to $-8 \%$ in $V$, which he identified as being due to cyclotron emission. Chanmugam \& Wagner (1977) described this "Remarkable System" consisting of a magnetic white dwarf (WD) accreting matter from a red dwart Martino 1995; Silber et al. 1996), which is sufficiently strong so the accretion stream follows the magnetic field lines to the surface of the WD. Since its discovery, AM Her has become the prototype polar.

AM Her was observed in various modes of the mass accretion (a single-pole versus a two-pole accretion (Heise et al. 1985; Matt et al. 2000). The optical longterm activity of AM Her is dominated by alternating high and low states of brightness (e.g. Hudec \& Meinunger 1976; Feigelson, Dexter \& Liller 1978; Wu \& Kiss 2008; Kafka \& Hoard 2009)

Further observations of AM Her at $4.9 \mathrm{GHz}$ using the VLA by Chanmugam \& Dulk (1982) resulted in the first radio detection of an MCV. A flux density of $0.67 \mathrm{mJy}$ was observed. Subsequent VLA observations by Dulk et al.(1983) again detected radio emission from AM Her at $4.9 \mathrm{GHz}$ and gave upper limits at 1.4 and $15 \mathrm{GHz}$. More recent radio observations of AM Her have yielded similar fluxes (Mason and Gray, 2006) and highly sensitive Jansky VLA observations indicate that AM Her is a persistent

radio source (Barrett et al. 2017\}.
Using the Na i absorption lines and noting the lack of TiO band heads, allowed Young and Schneider (1979) to establish the donor star to be an M4-M5V type. In order to investigate emission from the white dwart, the continuum in features in the white dwarf spectrum of AM Her (Paerels et al. (1996). Mauche \& Raymond (1998), consistent with this temperature.

\section{CRTS PHOTOMETRY}
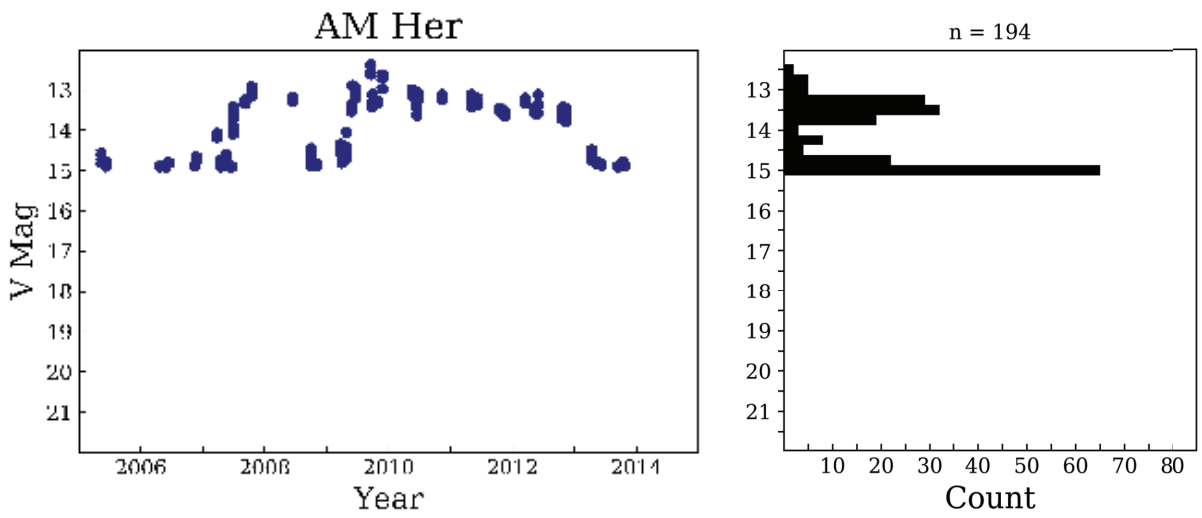

Figure 3: AM Her page. Each entry has a detailed data page and a brief written summary. These pages are under construction. Each entry has a page tailored to the individual binary. 
individualized data sheet for each MCV. The first level database, see Figure 1, is constructed as follows. The variable star name, e.g. AM Her, is given along with the discovery name, e.g. $3 \mathrm{U}$ 1809+50. Equatorial coordinates are downloaded from Gaia are listed. Entry are identified as being a polar or IP. The orbital periods and the white dwarf spin periods are listed. Some IPs have been described as Low Luminosity IPs and we identified these as LIPs. We also introduced a new group of IPs that we call transitional IPs labeled as TIPs. These are IPs that are best described as IPs evolving into polars. A few MCVs, of both types have experienced a classical novae explosion; e.g. GK Per, DK Her, and V1500 Cyg. If so, the novae name, e.g. Novae Per 1904 is also listed on the main data table. Other information, radio detection and so on, are listed. A finder chart is provided as well as ROSAT image of the field. Other data will be included as they become available. An extended data page is linked to the entries in Figure 3. These pages are not complete in the initial release version. Basic information is given, but information for each entry will increase and the number of sources will increase; as is the essence of the 'living' catalog. The catalog will develop along with the growth of the field.

Each source page has a finder chart from the Digitized Sky Survey, a Rosat image, as well as equatorial, galactic, and ecliptic coordinates. Distance estimates from Gaia (DR2) using an uncertainty model [1] are given. Eventually It will include light curves made from publicly available data and other details from public databases. Some data analysis such as period detections and magnetic field measurements will be provided in some cases. There will be a brief written summary of the binary as well as links to ADS references as well as the source link in Simbad. The goal of MCVs-Catalog is to provide quick access to data from the literature and as well as public sources of data on polars and intermediate polars for access to researchers.

\section{References}

[1] Bailor-Jones et al., 2018, AJ, 156, 58.

[2] Wolf, M. 1924, Astronomische Nachrichten (in German), 220, 255.

[3] Tapia, S. 1977, ApJ (Lett), 212, L125.

[4] Giaconni, R., 1974, ApJ Supp. 27, 37.

[5] Hearn, D. R., et al., 1976, ApJ (Lett), 203, L21.

[6] Chanmugam, G. \& Wagner, R. L. 1977, ApJ (Lett), 213, L13.

[7] Gänsicke, B. T.; Beuermann, K.; de Martino, D., 1995, A\&A, 303, 127

[8] Silber, A., et al., 1996, ApJ, 460, 939.

[9] Heise et al. 1985, A\&A, 148 L14.

[10] Matt, G., et al., 2000, A\&A, 358, 177.

[11] Hudec, R. \& Meinunger, L. 1977, Mitt. Ver. Sterne, 8, 10.

[12] Feigelson, E., Dexter, L., \& Liller, W., 1978, ApJ, 222, 263.

[13] Wu, K. \& Kiss L. L., 2008, A\&A, 481, 433.

[14] Chanmugam, G. \& Dulk, G. A., 1982, ApJ (Lett), 255, L107. 
[15] Dulk G. A., Bastian, T. S., \& Chanmugam, G. 1983, ApJ, 273, 249

[16] Mason, P. A. \& Gray, C. L., 2007, ApJ, 660, 662.

[17] Barrett, P., et al. 2017, ApJ, 154, 252.

[18] Young, P. J., \& Schneider, D. P. 1979, Ap. J., 230, 502.

[19] Paerels F. et al., 1996, ApJ, 464, 884.

[20] Mauche, C. W., \&Raymond, J. C., 1998, ApJ, 505, 869. 